\title{
Performance Optimization of WSN Using Deterministic Energy Efficient Clustering Protocol: A Review
}

\author{
Rajesh Chaudhary, Dr. Sonia Vatta \\ 1. (STUDENT) School of CSE Bahra University, Shimla Hills Himachal Pradesh, INDIA \\ 2. (GUIDE) School of CSE Bahra University, Shimla Hills Himachal Pradesh, INDIA
}

\begin{abstract}
DEC (Deterministic Energy Efficient Clustering) protocol is dynamic, distributive, self-organizing and more efficient in terms of energy than any other of the existing protocols. DEC uses an easy approach which reduces computational overhead-cost to self-organize the sensor network.

A WSN is a specialized wireless network made up of large number of sensors and at least one base station in order to collect data or information from the fields where they are deployed. The foremost difference between WSN and the traditional wireless networks is that sensors are extremely sensitive to energy consumption. Energy saving is a vital issue while designing the wireless sensor networks [12]. The critical point of these sensor nodes is that they generally consume much energy while making communications and reduce the battery and network lifetime. So it is essential to design effective and energy aware protocols in order to enhance the life time of the network. In this paper, DEC (Deterministic Energy Efficient Clustering) protocol is described in order to improve the energy efficiency of the wireless sensor networks.
\end{abstract}

Keywords: - Wireless sensor network, clustering, heterogeneity, DEC, LEACH, energy.

\section{INTRODUCTION}

Deterministic Energy-efficient Clustering protocol (DEC), a deterministic model developed by using clustering scheme to manage and organize the WSN with mobile scenario. DEC protocol guarantees a better election of cluster-heads based on residual energy information. It is more energy efficient than LEACH (Low Energy Adaptive Clustering Hierarchy) and any other existing energy protocols.

Deterministic Energy-Efficient clustering protocol uses residual energy of each node in the cluster for election process or selection of $\mathrm{CH}$ (Cluster Head). However, the uncertainties in the cluster-head elections have been minimized in DEC. The setup phase used in LEACH protocol is modified, but the steady-state phase is kept same as that of in LEACH protocol. The $\mathrm{CH}$ election process is reorganized by using the remaining energy (RE) of each node as node's energy generally determined a priori. In DEC, the sink or Base Station (BS) generally elects $\mathrm{N}_{\mathrm{opt}}$ cluster-heads at round $\mathrm{m}$ for the network. The BS can only take part in the election of $\mathrm{CHs}$ if and only if $\mathrm{m}=1$.

One major concern of energy constrained on WSN is to design energy efficient communication protocol. Clustering the entire network is an efficient solution to increase WSN life time. This work proposes a clustering based communication protocol for periodical data gathering application in wireless sensor network. It employs the scheduled rotation of cluster heads, which removes the problem with the variability of the number of clusters generated in the dynamic, distributed and randomized protocol. DEC protocol shows more energy efficiency by only triggering the cluster formation when energy level of cluster heads falls below a certain threshold.

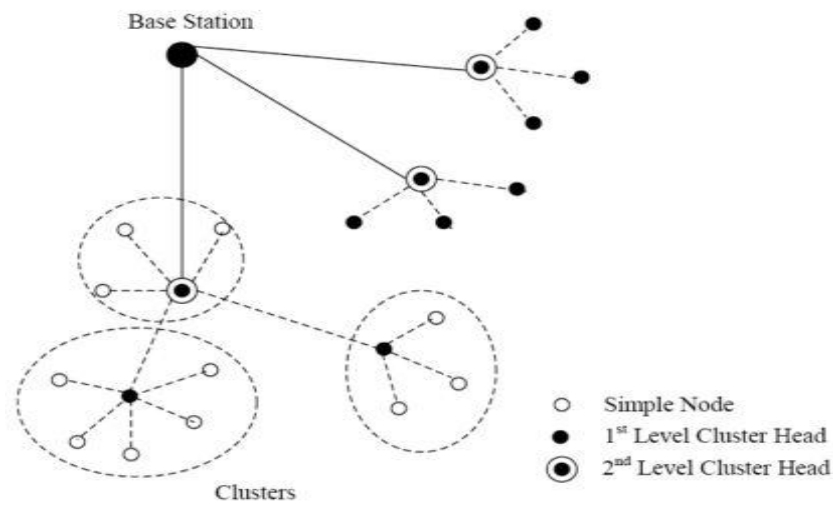

Figure 1: Hierarchical Cluster-Based Model 
A wireless sensor network (WSN) is often outlined as a network consisting of low-size and lowcomplex devices referred as sensor nodes that may sense the environment or surroundings and gather the knowledge from the observance field and communicate through wireless links; the information which is collected forwarded via multiple hops relaying to a base station or sink (also referred to as controller or monitor) that may use it domestically, or is connected to alternative networks [2]. Sensor nodes are typically spread out in a sensor field. Each of those scattered sensor nodes has the capabilities to gather information and route information back to the sink and also the end users. Information generally routed back to the end user by multihop infrastructure design through the sink. The sink could communicate with the task manager node via web/internet or Satellite [3].

\section{REVIEW OF LITERATURE}

Various researches done on the subject have been examined to find out the research questions. Also many books, articles, research papers etc. are studied where many researchers contribute their efforts. The detail is as given below:

W.R. Heinzelman, A.P Chandrakasan and H. Balakrishnan [5] projected LEACH protocol in 2000. LEACH is called as Low Energy Adaptive Clustering Hierarchy protocol. It is one of the mostly used hierarchical routing algorithms used in the sensor networks. The main plan of LEACH protocol is to divide the total wireless sensor network into many clusters. The cluster head node is randomly selected; the chance of every node to be selected as cluster head is equal attributable to which energy consumption of whole network is averaged. Thus LEACH will extend the network life cycle in WSNs.

Leach algorithm is cyclical; it provides a conception of round. Leach protocol runs with several rounds. Every round contains two states: cluster setup state and steady state. In the cluster setup state it forms cluster within the self-adaptive mode whereas in steady state it transfers the information.

The selection of cluster head depends on decision made 0 and 1 . If the number is smaller than that of the threshold value, the corresponding node becomes a cluster head for the current round.

The threshold is mostly given as:

$$
\mathrm{T}(\mathrm{n})= \begin{cases}\frac{\mathrm{p}_{1}}{1-\mathrm{p}^{*}(\mathrm{r} * \bmod 1 / \mathrm{p})} & \text { if } \mathrm{n} \varepsilon \mathrm{G} \\ 0 & \text { else }\end{cases}
$$

Where, $\mathrm{P}$ is the desired percentage of cluster heads (e.g. is like $3 \%$ or $4 \%$ ), $\mathrm{r}$ is that the current round, and $G$ is that the set of nodes that haven't been cluster heads within the last $1 / p$ rounds.

By considering this threshold, each node can be the cluster head at some point with $1 / \mathrm{p}$ rounds. Nodes that are cluster heads cannot become cluster head for the second-time for $1 / \mathrm{p}-1$ rounds. Therefore, most often every node has a $1 / \mathrm{p}$ probability of becoming cluster in every round. At the end of each round, every traditional or normal node that's not a cluster head choose the closest or nearest cluster head and joins that cluster to transmit the information. The cluster heads mix or combine and compress the information and forward it to the sink or the base station, hence extends the life of the most important or the major nodes. Problems in LEACH protocol are:

1) The cluster head node is randomly selected in LEACH protocol. There are some shortcomings attributable to the likelihood of every node to be selected as cluster head is same. After numerous rounds, the node with or containing greater remaining energy and the node with smaller remaining energy have same likelihood or probability to be chosen as cluster head. If the node which has smaller remaining energy is chosen as cluster head, it'll run out of the energy and die quickly, due to which network's robustness can be affected and life of the network becomes short.

S. Lindsey and C. Raghavendra [6] introduced Power Efficient Gathering in Sensor Information Systems (PEGASIS) protocol in 2002. It is an improved version of LEACH. Rather than forming clusters, it is based on forming chains of sensor nodes. One node is mainly responsible for routing the aggregated information to the sink. Every node in PEGASIS aggregates the collected information with its information, and then passes the collected data to the next ring. The distinction from LEACH is to employ or use multi hop transmission and selecting only one node to transmit to the sink or base station. Whereas the overhead caused by dynamic cluster formation is eliminated, multi hop transmission and data or information aggregation is employed or used, PEGASIS outperforms the LEACH. Problems in PEGASIS protocol are:

1. In PEGASIS sensor nodes usually or probably die early.

2. It is assumed that every sensor node is often able to communicate with sink directly; however nodes typically use multi-hop communication with the sink in practical cases. Moreover, long-range communication directly from the node to the sink will breed an excessive amount of energy consumption. 
3. The communication manner suffers from excessive delays caused by the one or single chain for distant nodes and a high probability for any node to become a bottleneck.

In 2001, A. Manjeshwar and D. P. Agarwal [7] proposed Threshold sensitive Energy Efficient sensor Network Protocol (TEEN) protocol. Nearer nodes form clusters, with a cluster head to transmit the collected information to one higher layer. Forming the clusters, cluster heads broadcast 2 threshold values. 1st one is hard threshold; it is minimum possible value of an attribute to trigger a sensor node. Hard threshold permits nodes to transmit the event, if the event happens within the range of interest. Thus a noteworthy reduction of the transmission delay happens. Unless an amendment of minimum soft threshold happens, the node doesn't send a new data packet. Soft threshold prevents from the redundant information/data transmission. Hence this is suitable for time-critical applications as this protocol is attentive to the rapid changes in the perceived attribute. Problems in TEEN are:

1. It is not appropriate for periodic reports applications since the user might not get any data at all if the values of the attributes may not arrive at the threshold.

2. If $\mathrm{CHs}$ don't seem to be within the communication range of each other, the data may be vanished, because information transmission is accomplished only at CHs.

A. Manjeshwar and D. P. Agarwal [8] projected Adaptive Threshold sensitive Energy Efficient sensor Network Protocol (APTEEN) protocol in 2002. The protocol is a modification of TEEN aiming to capture time-critical events and periodic data collections together. The network architecture is same as TEEN. While forming clusters, the cluster heads circulate attributes, the threshold values, and therefore the transmission schedule to any or all nodes. Cluster heads are also responsible for data aggregation so as to decrease the size of data transmitted and the energy consumed. According to energy dissipation and network lifetime, TEEN provides higher performance than LEACH and APTEEN because of the reduced number of transmissions. The main shortcomings of TEEN and APTEEN are overhead and complexity of forming clusters in multiple levels, implementing threshold-based function etc. APTEEN is based on query system which permits 3 types of queries: historical, on-time, and persistent which can be employed in hybrid network. Problems in APTEEN protocol are:

1. There exist supplementary complexity which is required to implement threshold functions and the count time.

2. Actually, both TEEN and APTEEN have the identical drawbacks of additional overhead and complexity of cluster construction in multiple levels.

One another research by G. Smaragdakis, I. Matta and A. Bestavros in 2004 has been found that [9] Stable Election Protocol (SEP) protocol is a further modification to the LEACH protocol. It's heterogeneous aware protocol, supported weighted election probabilities of every node to become cluster head according to their specific energy. This approach certifies that the cluster head election is arbitrarily selected and distributed based on the fraction of energy of every node assuring a uniform use of the nodes energy. In this protocol, 2 types of nodes (two tier in-clustering) and 2 level hierarchies were considered. The shortcoming of SEP method is that the election of the cluster heads among the two type of nodes is not dynamic, which results that the nodes which are far away from the powerful nodes will die first.

In 2005, M. Ye, C. Li, G. Chen and J. Wu [10] introduced Energy Efficient Clustering Scheme (EECS) protocol. It is a novel clustering scheme for periodical data collecting applications for wireless sensor networks. It elects cluster heads with more remaining energy through local radio communication. In the cluster head election phase, a stable number of candidate nodes are elected and compete for cluster heads according to the node residual energy. The competition method is localized without iteration. The process also produces a near uniform distribution of cluster heads. Moreover in the cluster formation phase, a unique approach is introduced to balance the load among cluster heads. However, on the other hand, it will increase the necessity of global knowledge regarding the distance between the cluster-heads and the base station. Limitations of EECS are:

1. On account of single-hop communication in EECS, long-range transmissions directly from $\mathrm{CHs}$ to the BS may lead to huge energy consumption. Thus, it is not suitable for large-range networks.

3. EECS produces rather more control overhead complexity because all nodes must compete for becoming $\mathrm{CHs}$.

In 2006, Q. Li, Z. Qingxin and W. Mingwen [11] designed Distributed Energy Efficient Clustering Protocol (DEEC) protocol. DEEC protocol is a cluster based method for multi level and 2 level energy heterogeneous wireless sensor networks. In this scheme, the cluster heads are chosen using the probability based on the ratio between residual energy of every node and the average energy of the network. The era of being cluster-heads for nodes are entirely different according to their initial and residual energy. The nodes with more initial and remaining energy have greater chances of the becoming cluster heads compared to nodes with low energy. Disadvantages of DEEC:

1. Advanced nodes always punish in the DEEC, particularly when their residual energy reduced and when they come in the range of the normal nodes. During this position, the advanced nodes die rapidly than the others. 
O. Younis and S. Fahmy projected [4] Hybrid Energy Efficient Distributed clustering Protocol (HEED) protocol in 2004. It extends the fundamental or the basic scheme of LEACH by using residual energy as primary parameter and network topology features such as node degree, distances to neighbours are only used as secondary parameters to shatter the tie between the candidate cluster heads, as a metric for cluster choice to attain power balancing. The clustering process is split into a number of iterations, and in every iteration nodes that are not covered by any cluster head doubles their probability of becoming a cluster head. As these energyefficient clustering protocols further enables each node to probabilistically and independently decide its role in the clustered network. Moreover it cannot guarantee optimal elected set of cluster heads. Limitations in HEED protocol:

1. Similar to LEACH, the clustering in each round imposes significant overhead in the network. This overhead causes remarkable energy dissipation which results in decreasing the network lifetime.

2. HEED suffers from a subsequent overhead since it needs several iterations to form the clusters. Therefore at iteration, a lot of packets are broadcasted.

3. Some CHs, particularly near the sink, might die earlier because these $\mathrm{CHs}$ have huge workload.

Harneet Kour and Ajay K. Sharma,[2] 2010 described about the H-HEED protocol. This protocol is basically used in heterogeneous wireless sensor network. H-HEED protocol is employed to extend the network life [2]. The impact of heterogeneity in terms of node energy in wireless sensor network has been stated. HHEED (Heterogeneous Hybrid Energy Efficient Distributed) is the revised version of the HEED protocol in terms of non- homogeneity. Here the cluster head is chosen based on the fraction of residual energy to the utmost energy possessed by the sensor nodes. Head to head communication takes place and unlike energy leveled networks have been formed. The energy efficiency has been verified in terms of the energy needed for the transmission and reception of the data. Here the node substitution takes place in order to reenergize the network and to enhance the network life. Disadvantage of H-HEED is H-HEED can't work or out perform well in the homogenous environment as DEC protocol can do so.

\section{PROBLEM DEFINITION}

The problem to be worked upon will be formed from the following facts. Wireless sensor network using nonclustering approach [4] is shown as:

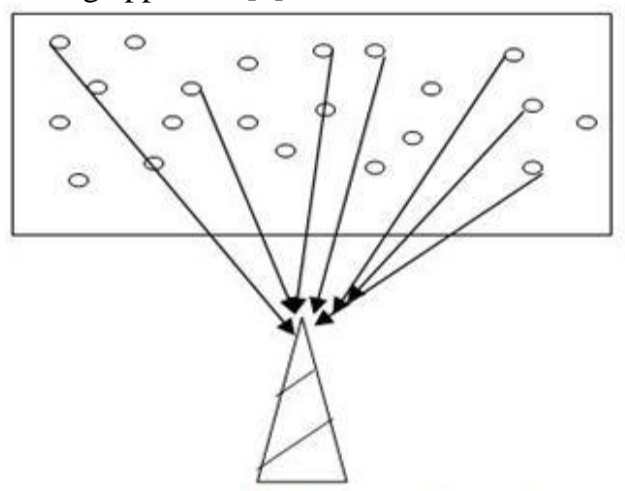

(a) Single-hop without clustering.

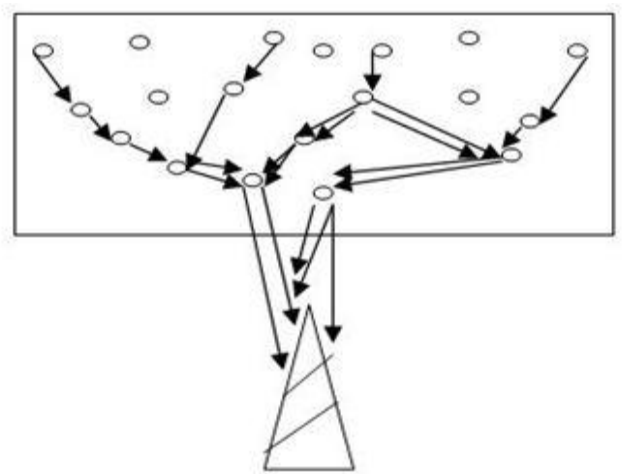

(b) Multi-hop without clustering.

Figure 2: Sensor forwarding information without clustering and aggregation [4].

Figure 2: (a) Shows Single hop case where each node transmits its frame directly to the sink node or to the base station. Single-hop without clustering means it does not use the clustering approach to aggregate the data. Therefore, it consumes more energy while sending data to the base station or to the sink.

Figure 2: (b) Shows Multi hop case where in each path, $N$ sensor nodes are involved Each node has several parameters but the most significant parameters in our analysis are node remaining energy, throughput and transmission power. Packet of size $Q$ is going to be transmitted from the sensor to sink with a given end-to-end latency constraint. Multi-hop without clustering means it uses multiple paths for communication but does not use the clustering approach to aggregate the data for energy efficiency [4].

Single-hop routing, can be much more energy efficient than multi-hop routing. Besides energy efficiency, single-hop routing can also have advantages such as end-to-end delay, lower packet loss parameters etc. [3].The main limitation of non-clustering approach is that it does not reduce energy consumption and it does not improve the energy efficiency of the network and furthermore end to end delay is more in non clustering approach as one node is involved in communication of other nodes in case of multihop without clustering and routing overhead also increases. Thus to overcome the problems of non-clustering approach, clustering approach in Deterministic energy efficient clustering protocol will be applied. 


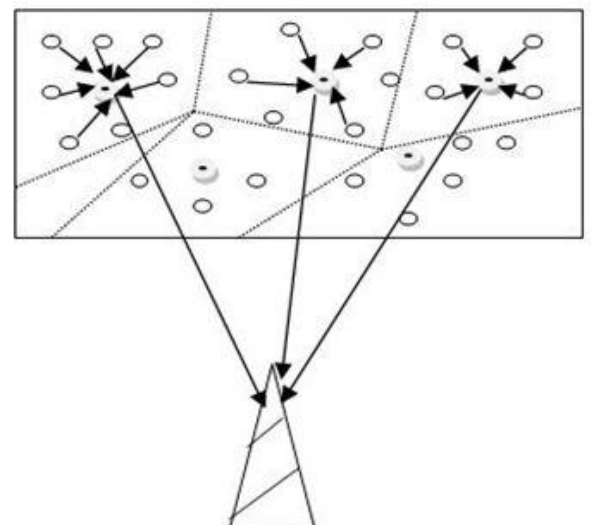

(a) Single-hop with clustering.

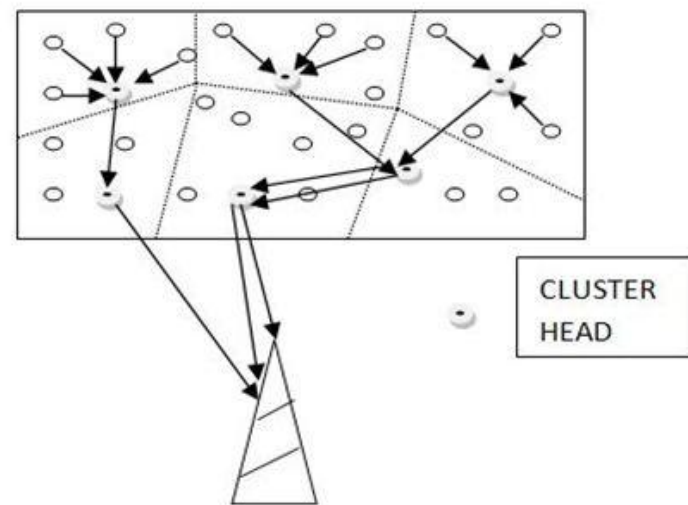

(b)Multi- hop with clustering

Figure3: Sensor forwarding information with clustering and aggregation [4].

The essential operation in sensor node clustering is to select a set of cluster heads from the set of nodes deployed in the network, and to cluster the remaining nodes with these cluster heads. Cluster heads are further responsible for creating coordination among the nodes within their clusters and aggregation of their data and communication with each other and/or with external observers on behalf of their clusters.

Figure 3: Depicts an application where sensors periodically transmit information to a remote observer. The figure illustrates that clustering can reduce the communication overhead for both single-hop and multi-hop networks. Periodic re-clustering can select nodes with higher residual energy to act as cluster heads.

Network lifetime is prolonged through (i) reducing the number of nodes contending for channel access, (ii) summarizing information and updates at the cluster heads, and (iii) routing through an overlay among cluster heads, which has a relatively small network diameter[4].

\section{PROPOSED WORK}

The proposed work consists of technique to be used in order to optimize the performance of wireless sensor networks.

\section{TECHNIQUE TO BE USED:}

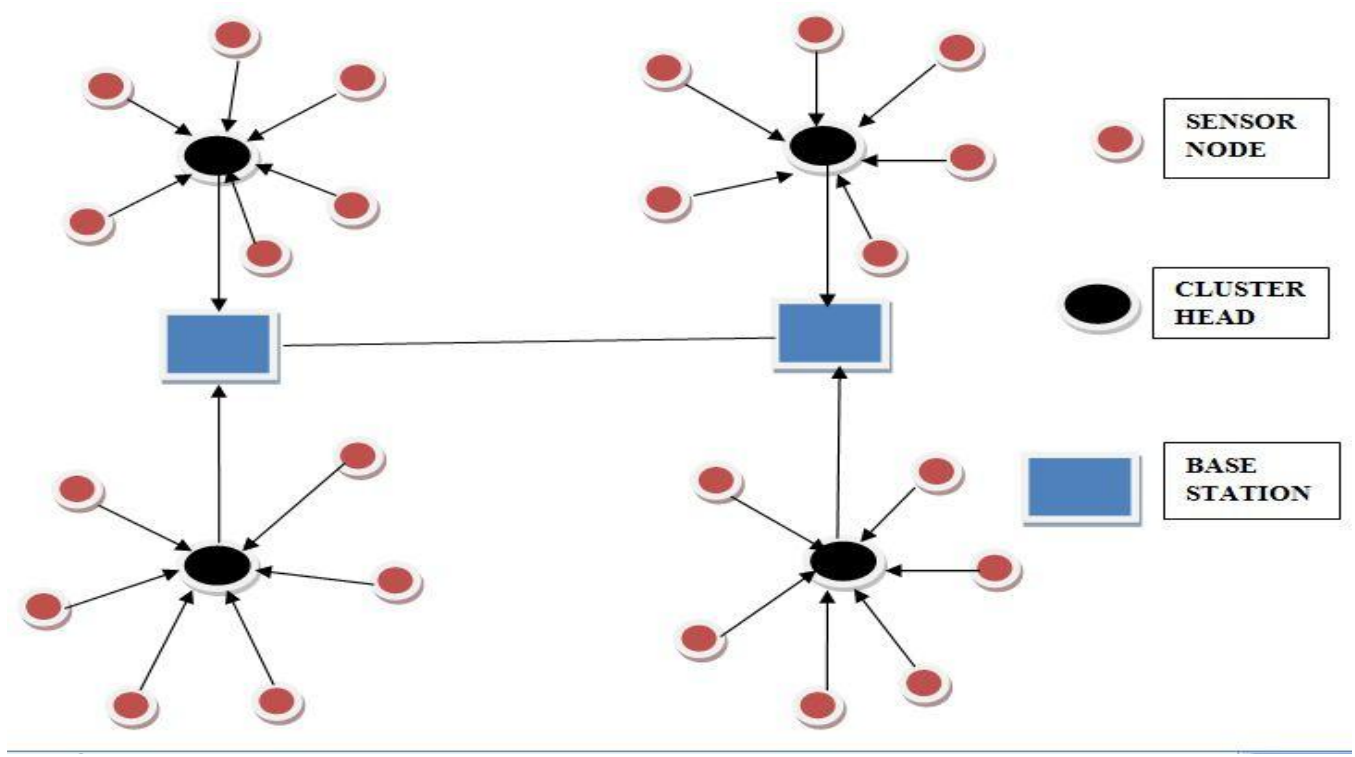

Figure 4: Scenario Case Diagram

Deterministic Energy-Efficient Clustering Protocol (DEC):

Deterministic Energy-Efficient clustering protocol uses residual energy of each node in the cluster for election process or selection of $\mathrm{CH}$ (Cluster Head). DEC [17] seems to be similar to an ideal solution as shown in Figure 5. 


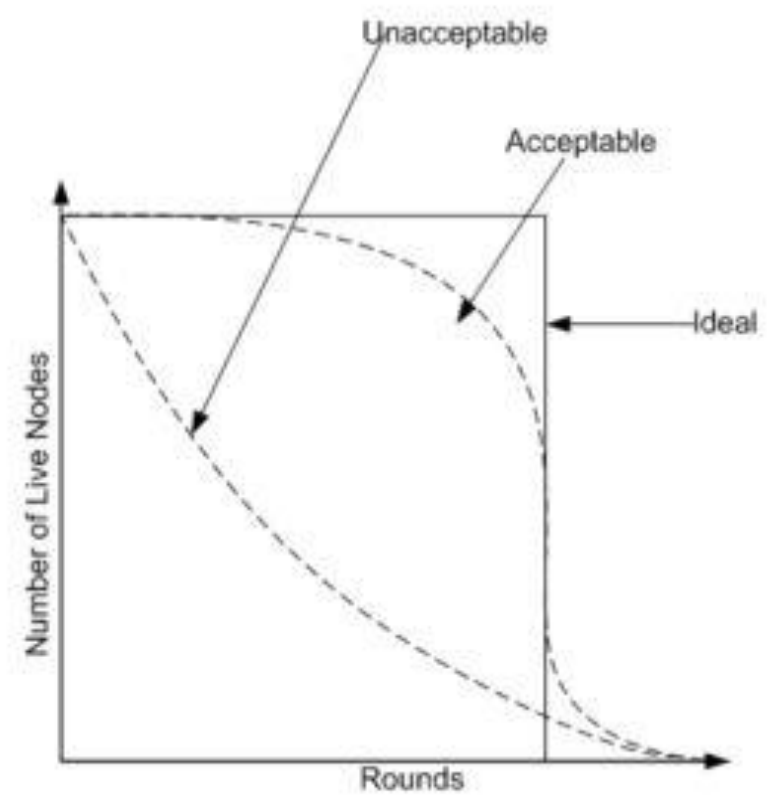

Figure 5: Behavior of node energy consumption overtime

However, the uncertainties in the cluster-head elections have been minimized in DEC. The setup phase which is utilized in LEACH (Low Energy Adaptive Clustering Hierarchy) protocol is further modified, but the steady-state phase is kept similar as that of in LEACH protocol. The $\mathrm{CH}$ election process is reorganized by using the remaining energy (RE) of each node as node's energy generally determined a priori. In DEC, the sink or Base Station (BS) generally elects $\mathrm{N}_{\text {opt }}$ cluster-heads at round $\mathrm{m}$ for the network. The BS can only take part in the election of $\mathrm{CHs}$ if and only if $\mathrm{m}=1$.

The elected CHs advertise their role using CSMA MAC just as in LEACH. However, in DEC unlike in LEACH, the join-request message will contain CM-ID, CH-ID, CM-RE (cluster member-residual energy) and the header that indicates it as a request. This way the RE information of CMs is known to their respective CHs thus localized and it can be utilized for $\mathrm{CH}$ rotation in the subsequent rounds. After the setup phase ends, the steady phase begins, but before the end of this phase, the current CHs further checks the CM-RE's information received to decide whether they will remain as $\mathrm{CHs}$ or relinquish their roles by choosing any node in their clusters with the highest RE as the new CHs.[17] After this, decision is made for the new CHs, all the data from the current round is communicated to the BS, the current round $(\mathrm{r}=\mathrm{m})$ ends (therefore a perfect synchronization is assumed in DEC, same as LEACH).

The next round $\mathrm{r}=\mathrm{m}+1$ begins; but since the new CH's are already chosen in the preceding round therefore they broadcast their role in the new round, Cluster members (CMs) join their cluster as mentioned earlier in the above paragraph. Therefore now steady phase begins again. This process continues for each round till the last node dies.

With this method, the battery life of WSNs is significantly optimized. Based on various studies, the followings facts are observed, which makes the DEC protocol desirable:

1) The $\mathrm{CH}$ election is locally decided based on each node's remaining energy (RE). In DEC, each round is independent of the subsequent round which is much distinct from LEACH etc.

2) DEC assures every node a chance of election as cluster-head as long as it's RE is higher than its neighbors.

3) DEC ensures a fixed $N_{\text {opt }}$ cluster-head is chosen.

4) DEC also minimizes the overhead cost of computation associated with $\mathrm{CH}$ search in the existing protocols, by refining this search to cluster $N_{i}$ at round $\mathrm{m}$.

5) DEC assures that each $\mathrm{CH}$ has enough energy to take up its role, until at least the end of the network lifetime, unlike in LEACH.

In Figure 4 Scenario DEC routing protocol is implemented in order to work in heterogeneous environment. In DEC protocol, using clustering approach there are two base stations in two different networks.

These Base Stations are connected to Cluster Head's for the communication purpose. In wireless sensor network, cluster is a group of sensor nodes in which one node acts as a cluster head and remaining nodes acts as member nodes $[15,3]$.Cluster head is selected based on the fraction of residual energy to the maximum energy possessed by the sensor nodes. In DEC protocol, head to head communication takes place. Also node replacement takes place in order to re-energize the network and to increase the network lifetime $[2,13$, 
14].Clustering helps in reducing the member of exchanged communication in wireless sensor network resulting in low consumption of battery power of individual sensor nodes. This increases the life span of the wireless sensor network $[15,16]$.

\section{CONCLUSION}

This work illustrates about the wireless sensor networks. Sensors in the sensor field are generally deployed in order to sense the data or to gather the information which is further send to the sink so as to transfer the data through internet or satellite to the user end.

In this paper, DEC protocol is also described for heterogeneous wireless sensor network. DEC protocol is a more efficient protocol then all the other existing protocols which communicate through head to head node. The cluster selection approach has been used for providing efficient communication in the wireless networks. It also re-energized the network to improve the overall performance of the wireless sensor network. The overall energy required for transmission and reception of data is formulated by source and sink. The clustered formation is used for avoiding the number of unwanted static channels and to remove the redundant data information. Also wireless sensor networks without clustering approach has been described for single hop and multi-hop cases. These both cases generally do not reduce the energy consumption whereas increase routing or energy overhead. Also in case of multi-hop end to end delay time increases as each node has the possibility of being part of several paths at the same time. Therefore these nodes will consume more energy per unit of time than others. So, to overcome the limitations of non-clustering approach generally the deterministic energy efficient clustering protocol is used.

This work will help researchers to investigate further in the field and make more developments so as to optimize the performance of wireless sensor networks.

\section{REFERENCES}

[1] Wiley, 2010] Fundamentals of Wireless Sensor Networks - Theory and Practice

[2] Harneet Kour and Ajay K. Sharma, "Hybrid Energy Efficient Distributed Protocol for Heterogeneous Wireless Sensor Network", International Journal of Computer Applications (0975 - 8887) Volume 4 No.6, July 2010.

[3] I.F. Akyildiz, W. Su*, Y. Sankarasubramaniam, E. Cayirci, "Wireless sensor networks: a Survey", Broadband and Wireless Networking Laboratory, School of Electrical and Computer Engineering, Georgia Institute of Technology, Atlanta, GA 30332, USA.

[4] Ossama Younis and Sonia Fahmy. 2004. Distributed Clustering in Ad-hoc Sensor Networks: A Hybrid, EnergyEfficient Approach. In Proceedings of IEEE INFOCOM, Hong Kong, an extended version appeared in IEEE Transactions on Mobile Computing, 3(4)

[5] W. Heinzelman, A. Chandrakasan and H. Balakrishnan. 2000. Energy-Efficient Communication Protocol for Wireless Microsensor Networks. Proceedings of the $33^{\text {rd }}$ Hawaii International Conference on System Sciences (HICSS '00).

[6] S. Lindsey, C. Raghavendra. 2002. PEGASIS: Power Efficient Gathering in Sensor Information Systems. IEEE Aerospace Conference Proceedings, Vol. 3, 9-16 pp. 11251130.

[7] A. Manjeshwar and D. P. Agarwal. 2001. TEEN: a routing protocol for enhanced efficiency in wireless sensor networks. In 1st International Workshop on Parallel and Distributed Computing Issues in Wireless Networks and Mobile Computing.

[8] A. Manjeshwar and D. P. Agarwal. 2002. APTEEN: A hybrid protocol for efficient routing and comprehensive information retrieval in wireless sensor networks. Parallel and Distributed Processing Symposium, Proceedings International, IPDPS, pp. 195-202.

[9] G. Smaragdakis, I. Matta, A. Bestavros. 2004. SEP: A Stable Election Protocol for clustered heterogeneous wireless sensor networks. In Second International Workshop on Sensor and Actor Network Protocols and Applications (SANPA).

[10] M. Ye, C. Li, G. Chen, J. Wu. 2005. EECS: an energy efficient cluster scheme in wireless sensor networks. In IEEE International Workshop on Strategies for Energy Efficiency in Ad Hoc and Sensor Networks (IEEE IWSEEASN-2005), Phoenix, Arizona, April 7-9.

[11] Q. Li, Z. Qingxin, and W. Mingwen. 2006. Design of a distributed energy efficient clustering algorithm for heterogeneous wireless sensor networks. Computer Communications, vol. 29, pp. 2230-7.

[12] K. Padmanabhan, Dr. P. Kamalakkannan "Energy Efficient Adaptive Protocol for Clustered Wireless Sensor Networks" IJCSI International Journal of Computer Science Issues, Vol. 8, Issue 5, No 1, September 2011 ISSN (Online): 1694-0814

[13] ManjuBala and LalitAwasthi, "Proficient D-HEED Protocol for Maximizing the Lifetime of WSN and Comparative Performance Investigations with Various Deployment Strategies”, International Journal of Advanced Science and Technology Vol. 45, August, 2012. 
[14] S. Karthikeyan and S. Jayashri, "Energy Efficient System for Heterogeneous Wireless Sensor Network", European Journal of Scientific Research ISSN 1450-216X Vol.72 No.4 (2012), pp. 599-607, (C) Euro Journals Publishing, Inc. 2012.

[15] Bhawnesh Kumar and Vinit Kumar Sharma, "Distance based Cluster Head Selection Algorithm for Wireless Sensor Network”, International Journal of Computer Applications (0975 - 8887) Volume 57No.9, November 2012.

[16] RaziehSheikhpour, Sam Jabbehdari, Ahmad Khadem-Zadeh, " Comparison of Energy Efficient Clustering Protocols in Heterogeneous Wireless Sensor Networks" International Journal of Advanced Science and Technology Vol. 36, November, 2011.

[17] Aderohunmu F.A., Deng J.D., Purvis M.K., "A deterministic energy-efficient clustering protocol for wireless sensor networks", Proceedings of Seventh IEEE International Conference on Intelligent Sensors, Sensor Networks and Information Processing, pp.341 - 346, 2011. 